\title{
Lumen
}

Selected Proceedings from the Canadian Society for Eighteenth-Century Studies

\section{Voltaire et le théâtre comique : étaient-ils incompatibles?}

\section{Georges L. Bérubé}

Volume 15, 1996

URI : https://id.erudit.org/iderudit/1012470ar

DOI : https://doi.org/10.7202/1012470ar

Aller au sommaire du numéro

Éditeur(s)

Canadian Society for Eighteenth-Century Studies / Société canadienne d'étude du dix-huitième siècle

ISSN

1209-3696 (imprimé)

1927-8284 (numérique)

Découvrir la revue

Citer cet article

Bérubé, G. L. (1996). Voltaire et le théâtre comique : étaient-ils incompatibles? Lumen, 15, 17-26. https://doi.org/10.7202/1012470ar d'utilisation que vous pouvez consulter en ligne.

https://apropos.erudit.org/fr/usagers/politique-dutilisation/ 


\section{Voltaire et le théâtre comique: étaient-ils incompatibles?}

Ce n'est pas sans un certain étonnement que les critiques ont constaté, au fil des années, le paradoxe que suggère le titre de cette communication. En effet, comment expliquer que l'auteur d'innombrables saillies, épigrammes et satires, le créateur de personnages comiques comme la vieille à la fesse coupée ou les Oreillons anthropophages qui se réjouissent à l'approche du festin en hurlant: 'mangeons du jésuite, mangeons du jésuite, ${ }^{1}$ ou encore cette majestueuse Jeanne la Pucelle, qui chevauche sur son âne à la rescousse du royaume de France, ${ }^{2}$ qu'un tel écrivain, disions-nous, ait manqué de vis comica au point que son théâtre comique est mort et enterré depuis bientôt deux siècles? Ronald Ridgeway résumait bien la situation:

The comedies seem to have disappeared without trace. Of the many paradoxes of Voltaire's literary career, not the least extraordinary is the fact that one of the wittiest and most amusing of all writers, and one moreover who was an accomplished actor, director, and playwright, failed dismally in comedy. [...] his comedies are even less comic than his tragedies are tragic. ${ }^{3}$

Par surcroît, songeons que l'homme lui-même, reconnu pour sa conversation brillante et pleine d'esprit - n-a-t-on pas prétendu qu'il incarna la quintessence de l'esprit français du XVIIIe siècle? - nous a laissé une volumineuse correspondance remplie d'éléments drôles et de récits comiques. Cette propension au comique se retrouve même dans le Dictionnaire philosophique, texte sérieux par excellence, comme arme dans la guerre contre l'Infame. Qui d'autre qu'un Voltaire aurait eu l'audace d'inclure un article comme 'Baiser' dans une oeuvre de propagande philosophique?

J'en demande pardon aux jeunes gens et aux jeunes demoiselles, mais ils ne trouveront point ici peut-être ce qu'ils chercheront. Cet article n'est que pour les savants et les gens sérieux, auxquels il ne convient guère. [...] On connaît le chapitre sur les baisers, dans lequel Jean de La Casa, archevêque de Bénévent, 
dit qu'on peut se baiser de la tête aux pieds. Il plaint les grands nez qui ne peuvent s'approcher que difficilement; et il conseille aux dames qui ont le nez long d'avoir des amants camus. ${ }^{4}$

Malgré tout cela, les critiques sont à peu près unanimes à juger le théâtre comique de Voltaire un échec lamentable. Sommes-nous donc justifiés d'avancer qu'il y aurait eu entre les deux incompatibilité?

Précisons, en premier lieu, qu'il serait impossible de traiter de façon exhaustive, dans le cadre d'une étude aussi brève que celle-ci, un sujet complexe. En nous limitant donc aux deux premières tentatives de Voltaire dans le genre comique, soit L'Indiscret et La Fête de Bélébat qui datent toutes deux de 1725 , nous aimerions proposer quelques réflexions 1) sur les rapports que Voltaire entretenait avec ses oeuvres et 2) sur les rôles que le théâtre comique a pu jouer dans l'évolution de la pensée du dramaturge.

Partons d'abord de l'hypothèse que ce que La Harpe, dans son Cours de littérature ancienne et moderne, identifie comme étant la lacune qui a empêché Voltaire de réussir dans le genre comique, a été en réalité, non une lacune, mais un projet commun à toutes les oeuvres écrites par Voltaire à cette époque. La Harpe souligne notamment:

Dans une satire, dans une épître, dans un badinage quelconque, la gaieté naturelle et l'esprit peuvent vous suffire; vous parlez en votre nom, et vous pouvez vous servir de tous vos moyens. Mais au théâtre tout change de face: il faut d'abord être comique par les situations et les caractères, et Voltaire n'a jamis su être ni l'un ni l'autre [...]. En fait d'esprit, il était trop lui pour devenir un autre $[\ldots]^{5}$

Toujours selon La Harpe, cet homme qui 'a donné son esprit à tout un siècle' n'arrivait pas à s'oublier assez pour créer un personnage de comédie. Voilà un point capital sur lequel il faut se pencher un instant. $\mathrm{Si}$, effectivement, la tare relevée par le critique littéraire explique en partie l'échec du théâtre comique de Voltaire, nous voudrions proposer, à la lumière de tout ce qu'il écrit dans ces années-là, qu'il n'entendait nullement se plier à la tradition et aux règles du genre tel qu'on le pratiquait alors. Ne s'en prend-il pas aux auteurs à la mode lorsqu'il lance sa comédie de L'Indiscret?

Dancourt et Legrand ont accoutumé le parterre au bas comique et aux grossièretés, et insensiblement le public s'est formé le préjugé que des petites pièces en un acte doivent être des farces pleines d'ordures et non pas des comédies nobles ou les moeurs soient respectées. ${ }^{6}$ 
Voltaire, croyons-nous, entend faire des expériences formelles et adapter le genre comique pour qu'il devienne un véhicule efficace qui traduise sa pensée à lui.

En effet, c'est ce que Voltaire avait tenté dans Oedipe, sa première tragédie; choeurs à l'antique, présence d'un personnage nouveau et problématique dans un mythe bien connu, introduction de vers-chocs qui cristallisent la révolte des protagonistes contre la religion, etc.. Dans une recherche antérieure, nous avons étudié une de ces innovations, soit le personnage de Philoctète. ${ }^{7}$ Ainsi, nonobstant un vice de construction fondamental, Voltaire n'a jamais voulu remanier sa tragédie, pour la bonne et simple raison que, dans ce personnage, il avait investi une bonne part de sa propre subjectivité: l'apparition d'un personnage pur et innocent qui met en cause la corruption de la société française de la Régence. Le même phénomène se produit dans La Henriade dans le personnage de Henri IV. Ce roi venu d'ailleurs représente un élément perturbateur, étranger, dans le sens que de nouvelles valeurs, à savoir l'amour et surtout la tolérance religieuse, sont proposées à la société close et décadente des fils de Catherine de Médicis. Il appert donc que, dans la plupart des écrits du début de sa carrière, Voltaire cherche des formes susceptibles de lui permettre d'affirmer plus ou moins ouvertement un message radicalement différent. Pensons à l'audacieuse épître à Madame de Rupelmonde de 1722, connue aussi sous le titre d'Epîtreà Uranie ou Le Pour et le contre. Les premiers vers prouvent sans l'ombre d'un doute qu'ils sont le prolongement de ceux d'Oedipe:

Tu veux donc, belle Uranie,

Qu'érigé par ton ordre en Lucrèce nouveau,

Devant toi, d'une main hardie,

Aux superstitions j'arrache le bandeau [...].

Et le tout débouche sur une déclaration d'indépendance presque inouie pour l'époque:

Je ne suis pas chrétien; mais c'est pour t'aimer mieux. ${ }^{8}$

[mon Dieu]

En plus des genres canoniques, Voltaire a développé jusqu'au plus haut point le genre le plus personnel qui soit, la lettre. La diversité et la quantité de celles-ci témoignent d'une prédilection évidente. Nous souscrivons d'ailleurs à la thèse de Geneviève Haroche-Bouzinac qui considère la correspondance du jeune Voltaire comme un 'laboratoire' des oeuvres à venir: 
Les lettres du jeune Voltaire [...] ont [...] accompagné d'autres gestations. Elles deviennent le laboratoire des oeuvres en préparation, comme nous l'avons remarqué à propos de L'Indiscret et de Mariamne. Telle expression du sentiment amical ou amoureux voisine avec le ton de La Henriade. Telle lettre en vers et prose offre une partie qui deviendra "épître." De façon plus lointaine les tournures épigrammatiques et quelques aspects de la leçon morale des contes sont annoncés, tandis que plusieurs chroniques, et surtout la lettre sur le voyage en Hollande conduisent aux Lettres anglaises. [...] La correspondance se présente rapidement comme un champ d'expérimentation. ${ }^{9}$

De la lettre ainsi envisagée, de la recherche constante d'une forme qui exprime adéquatement son je profond ont surgi, selon nous, l'essai historique tel Charles XII et le chef-d'oeuvre qui intègre la lettre et l'essai: les Lettres philosophiques. Finalement, discours critique et récit fictif se marient parfaitement dans un nouveau genre, le conte philosophique, où Voltaire se sent tout à fait à l'aise d'inviter le lecteur de lire au-delà de l'histoire. C'est donc à l'intérieur de cette expérimentation en progression constante qu'il faut envisager les deux comédies de Voltaire.

L'Indiscret, écrit et représenté en 1725 , constitue une première tentative apparemment tardive pour un auteur qui, vu sa tournure d'esprit, aurait pu s'intéresser au genre beaucoup plus tôt. Sans doute voulait-il d'abord asseoir sa réputation comme écrivain sérieux - la chose est acquise après le succès d'Oedipe, de Mariamne et de La Ligue - , avant de se lancer dans un genre assez peu valorisé. Selon Lilian Willens, dans son livre Voltaire's Comic Theatre: Composition, Conflict, and Critics, L'Indiscret représente une petite pièce sans grande valeur originale: 'a one-act play in alexandrines, [...] written in the classical tradition of the comedies of the seventeenth century and the early eighteenth century, with emphasis placed upon character portrayals and the depiction of the mores of the times. ${ }^{10}$ Petite pièce dans le genre classique, par conséquent. L'affirmation de Willens a pourtant de quoi surprendre: en quoi une pièce en 21 scènes et un seul acte, avec personnages épisodiques qui paraissent au début et puis disparaissent, est-elle 'dans la tradition classique'? N'annonce-t-elle pas de loin le 'monstre' que sera Le Mariage de Figaro? Dès sa création en 1725, les critiques ont remarqué ses nombreux accrocs aux règles de la dramaturgie, comme par exemple Marais qui rapporte dans son Journal: 'Voltaire vient de donner une petite comédie de L'Indiscret [...]; on dit qu'il y a beaucoup d'esprit: cependant elle a déplu à la chambre basse [le parterre] qui y a trouvé peu de règles $d u$ théâtre, et à la chambre haute [les loges, la cour], qui s'y est trouvée trop bien dépeinte ${ }^{\prime 11}$ (nous soulignons). Son succès mitigé - six représentations à sa première saison à la Comédie française et vingt-trois seulement de 1725 à 1778 - renforce l'impression que, loin d'être une petite pièce 
classique, l'oeuvre comportait des éléments qui ont dérangé le public. En effet, nous retrouvons ici la même technique qui avait assuré une partie de la renommée d'Oedipe, ce que nous appelons des vers-chocs, mais ceux de la comédie n'ont plus pour cible la religion et, surtout, ne jouissent plus de la complicité du public irrevérencieux de 1718. De l'avis de Marais, Voltaire aurait réussi à aliéner même le public qu'il souhaitait le plus rejoindre, celui des loges. Et la raison en est assez claire: les vers chocs - d'ailleurs fort nombreux - s'en prennent à la cour et à ceux qui la fréquentent. Imaginons la réaction des gens à la mode qui se croyaient l'objet d'une attaque aussi dure:

Et [...] à la cour, mon fils, l'art le plus nécessaire

N'est pas de bien parler, mais de savoir se taire.

Ce n'est pas en ce lieu que la société

Permet ces entretiens remplis de liberté:

Le plus souvent ici l'on parle sans rien dire;

Et les plus ennuyeux savent s'y mieux conduire (M.2:248).

La libre expression, si chère à Voltaire, n'a bien entendu aucun droit de cité ici. Mais avant d'aller plus loin, résumons brièvement une pièce assez peu connue de nos jours.

Après la mise en garde de sa mère, Euphémie, qu'à la cour il ne faut surtout pas parler des femmes, Damis se vante d'avoir fait la conquête $d^{\prime}$ Hortense, une riche veuve qui

Craint surtout de se voir en spectacle à la cour,

Et d'être le sujet de l'histoire du jour (M.2:249).

Ignorant les conseils de sa mère et confiant d'avoir sous peu toutes les femmes à ses pieds, le jeune étourdi provoque le commandeur Trasimon en annonçant à qui veut l'entendre qu'il va épouser Hortense, la nièce du commandeur. L'indiscrétion est d'autant plus choquante, qu'elle se produit devant Clitandre, l'amant à qui Hortense vient de donner son congé. Bien pire, Clitandre devient, bien malgré lui, le confident de Damis lorsque ce dernier lui confie le portrait de sa maîtresse. En désespoir de cause, l'amant rejeté se tourne vers Pasquin, un valet particulièrement audacieux, pour qu'il provoque la rupture du nouveau couple. Le stratagème fonctionne à merveille, mais à la dernière minute Hortense, toujours éprise du jeune indiscret, veut l'éprouver une dernière fois. Masquée et sous l'apparence d'une amante de Damis, elle sonde ses véritables sentiments. Déçue par un Damis incorrigible jusqu'à la fin - mais ayant pu récupérer ses lettres et son portrait - , Hortense 
choisit de revenir à Clitandre. Damis, quant à lui, est humilié mais indompté, car son règne à la cour est loin d'être terminé.

Comme on a pu le constater, cette pièce met en scène un petit-maître tout à fait actuel en 1725, qui tentait de faire fortune dans le Paris agité de l'époque. Ainsi, très peu de distance séparait les personnages ridiculisés sur le théâtre et les spectateurs qui étaient censés en rire. Plus révélateur encore, le renversement de l'ordre typique de la comédie moliéresque, où les maîtres sont bernés mais rétablis dans leur pouvoir au dénouement, fonctionne ici de façon bizarre: Euphémie et Trasimon, du début à la fin, plaident pour un ordre menacé, mais jamais renversé, et c'est Damis, non les maîtres, qui possède tous les travers ridicules. Puis, comme le remarque Willens, Damis n'a pas de remords au dénouement; il se moque plutôt de sa défaite passagère en s'écriant: 'Juste ciel! désormais à qui peut-on parler?' Quant à la morale, douteuse - c'est le moins qu'on puisse dire - , elle revient au commandeur Trasimon, qui se déclare meilleur manipulateur que le jeune freluquet:

C'est moi seul, mons Damis, qui fais ce mariage.

Adieu: possédez mieux l'art de dissimuler (M.2:274).

Le dénouement, si l'on entend par là le rétablissment de l'ordre, n'en est pas un. Si Trasimon obtient gain de cause, il est bien évident que la société française, elle, va continuer de produire des petits-maîtres en quantité. A défaut d'être un monde à l'envers, celui de L'Indiscret se veut un reflet tout à fait fidèle, mais trop ressemblant à la réalité pour faire rire. Et, sans le recul nécessaire, nous savons qu'il est presque impossible de mettre les rieurs de son côté.

Revenons maintenant à La Harpe qui écrivait: 'au lieu de se déguiser [Voltaire] successivement sous plusieurs formes, selon la nature du personnage, il prenait pour tous un masque et une marotte: c'était Voltaire en habit de bal. ${ }^{12}$ La forte présence du dramaturge se fait sentir dans L'Indiscret, comme elle se fera sentir plus tard dans L'Envieux et L'Ecossaise, c'est-à-dire à chaque fois qu'il cherche à régler son compte à un ennemi. Dans L'Indiscret, la critique des petits-maîtres apparait dans presque tous les discours, celui de la mère, Euphémie, de Trasimon, de Clitandre, d'Hortense, voire de Damis lui-même, lorsqu'il juge ainsi la France au dénouement:

Oui, la mode fait tout, décide tout en France;

Elle règle les rangs, l'honneur, la bienséance,

Le mérite, l'esprit, les plaisirs (M.2:271). 
Or, si pour Voltaire cela constitue une dénonciation, pour Damis c'est un savoir qui va lui permettre de mieux jouer le jeu pour atteindre le pouvoir. Sur le plan dramaturgique par contre, la comédie est sacrifiée au profit d'un but qui lui est étranger.

Deuxième expérience, La Fête de Bélébat. Contrairement à L'Indiscret, cette pièce n'a jamais été montée au Théâtre français et n'a fait l'objet que d'une seule représentation en 1725 chez le marquis de Livry à son château de Bélébat. Dans l'avertissement de l'édition de Kehl, on peut lire:'Tous les vers, à beaucoup près, ne sont pas de M. de Voltaire et ceux qui lui appartiennent sont faciles à distinguer.' Louis Moland ajoute pourtant que 'l'ensemble de la composition est certainement de Voltaire.'

Pourquoi, est-on en droit de se demander, s'intéresser à un petit divertissement éphémère qui, de l'aveu de Voltaire lui-même, il faudrait avoir vu 'pour en bien sentir l'agrément: les projets et les préparatifs de ces divertissements sont toujours agréables, l'exécution rarement bonne, et le récit souvent ennuyeux'? (M.2:282) Mais voilà précisément l'intérêt: le texte de la pièce, tout court et tout fragmenté soit-il, nous parvient sous la forme d'une lettre adressée à Son Altesse Sérénissime Mademoiselle de Clermont, surintendante de la maison de la reine. Il s'agissait donc de réjouir Marie Leczinska, reine de France, par la lecture de ce récit, ${ }^{13}$ elle qui appréciait les oeuvres du dramaturge au point de l'appeler 'mon pauvre Voltaire' (Best. D253).

Cette comédie, si tant est que ce terme puisse désigner une oeuvre sans actes, ni scènes, comporte à peine deux situations: le couronnement du curé de Courdimanche, puis son agonie et la désignation de son successeur. Telle quelle, l'oeuvre squelettique offre peu d'intérêt pour qui cherche un texte fini, complet, et bien travaillé. En revanche, l'état fragmentaire, qui n'est pas sans rappeler ces esclaves de Michel-Ange qui tentent vainement de se dégager du marbre, exprime à l'état brut l'essence des aspirations et de la pensée de Voltaire à cette époque. Notons, d'autre part, que le texte est double: épistolaire, c'est le je de Voltaire qui écrit à une princesse, sa destinataire; et dramatique, c'est Voltaire jouant son propre personnage qui prend la relève. Voyons un peu de quoi il est question.

A l'arrivée du curé de Courdimanche, bon vivant qui aime le vin, la table, les vers et l'amour, l'assemblée chante ses louanges pendant qu'on l'installe sur un trône, une couronne de laurier pendue au-dessus de sa tête. Mais son règne ne dure pas puisqu'on évoque un accident qui le mène à son lit de mort: 
Ah! notre curé

S'est bien échaudé

Faisant la lessive

Ah! notre curé

Est presque enterré

Pour s'être échaudé (M.2:285)

Si la scène change, il en va de même de l'écriture. Nous assistons alors à une longue exhortation sous forme d'épître, prononcée sans doute par Voltaire lui-même, dans laquelle il invite le curé à faire une confession générale:

Si vous aviez été toujours homme de bien,

Un bon prêtre, un nigaud, je ne vous dirais rien:

Mais qui peut, entre nous, garder son innocence?

Quel curé n'a besoin d'un peu de pénitence? (M.2:286)

L'exhortation prend fin avec la recommandation suivante: il faut dicter un testament et choisir parmi les gens de la fête, un 'successeur qui soit digne de vous' (M.2:288). Le choix, orchestré par Voltaire auteur, fait sourire: 'Mon successeur sera Voltaire,/ Pour mieux me faire regretter,' à quoi le bedeau et le choeur répondent:

Que de tous côtés on entende

Le beau nom de Voltaire, et qu'il soit célébré.

Est-il pour nous une gloire plus grande?

L'auteur d'Oedipe est devenu curé (M.2:290).

La marquise de Prie offre alors une couronne de laurier au poète-prêtre 'en attendant, dit-elle, encor mieux' (M.2:290). Le choeur se réjouit de l'ère nouvelle qui commence: finies, la pénitence et l'abstinence, car les plaisirs et l'amour 'suivent Voltaire en ce jour.' La cérémonie se termine enfin par une série de compliments que Voltaire adresse à chaque participant de marque: la marquise de Prie, le comte de Clermont, le marquis de Livry, seigneur des lieux, le président Hénault, etc.. Puis le choeur reprend l'hymne à la liberté: la raison qui murmure est chassée pour faire place aux plaisirs.

Peut-on douter de la présence accablante de la subjectivité ici? $\mathrm{D}^{\prime}$ abord la lettre qui sert de véhicule au récit; puis la forme dramatique qui contient tant de discours hétérogènes, dont une exhortation sous forme d'épitre, ensuite toute une série de compliments, voilà autant d'élements qui mettent en scène un Voltaire auteur, puis un Voltaire 
personnage qui, une fois couronné, cède la parole à Voltaireamant/courtisan qui remercie chacun d'avoir participé, ou du moins consenti, à son couronnement. Ne voilà-t-il pas une pure comédie? En premier lieu, le renversement de l'ordre: ce curé épicurien qui s'arroge le rôle de libertin, qui accepte la couronne de laurier et 'tous ces éloges avec l'air d'un homme qui sait qu'il en mérite encore davantage' (M.2:282), suivi du rétablissement de l'ordre: Voltaire, couronné roiprêtre des libertins, inaugure un monde nouveau, avec des valeurs révolutionnaires. Si ici tout est ironie, on comprend quand même le désir de Voltaire d'imposer sa puissance.

Quelles différences y a-t-il entre les nouvelles valeurs du roi Voltaire et celles préconisées par Damis, le héros humilié de L'Indiscret? Les deux ne cherchent-ils pas à affirmer leur puissance?

Que conclure de tout ceci? Le passage du genre épistolaire où la suprématie du je est absolue à la comédie - où le je doit disparaître s'avère imparfait, puisque Voltaire ne réussit pas - du moins sur le plan dramaturgique - à modifier L'Indiscret suffisament pour qu'il serve de véhicule à ses idées, tout en demeurant comique. Dans La Fête de Bélébat, où le je est omniprésent dans de multiples discours, le comique peut reprendre son droit de cité. Ainsi, dans la mesure où la comédie au XVIIIe évolue dans un sens autre que celui qu'aurait voulu lui donner Voltaire, nous pouvons dire que oui, dans les premières comédies, il y a incompatibilité entre Voltaire et la comédie de l'époque. Par contre, ce ne sera pas le cas plus tard, lorsque Voltaire tente de s'adapter au goût du public en faisant représenter $L^{\prime} E n f a n t$ prodigue, Nanine et $L^{\prime} E$ cossaise. Par contre, la recette du théâtre didactique ou de la pièce à thèse - qu'on retrouve en germe dans $L^{\prime}$ Indiscret - va revenir en force dans son théâtre tragique et engagé, dans des oeuvres tardives comme Les Scythes, Les Guèbres et Les Lois de Minos.

GEORGES L. BÉRUBÉ

Université York

Notes

1 Voltaire, Candide ou l'optimisme, dans Oeuvres complètes, éd. Louis Moland. t.21 (Paris: Garnier, 1878) ch. 12 et 16. Toutes nos références aux oeuvres de Voltaire renvoient à cette édition.

2 La Pucelle, chant 2 (M.9:47 et sq.)

3 Ronald Ridgway, Voltaire and Sensibility (Montréal: McGill-Queen's, 1973) 197. 


\section{6}

Georges-L. Bérubé

4 Dictionnaire philosophique, art. 'Baiser' (M.17:527-28).

5 Jean François de La Harpe, Cours de littérature ancienne et moderne (Paris: Didot, 1870) 2:508.

6 Correspondence and Related Documents, éd. Th. Besterman, dans Oeuvres complètes de Voltaire t.85 (Genève et Banbury: The Voltaire Foundation, 1968) Best. D246.

7 Cf. 'Le Personnage de Philoctète dans l'Oedipe de Voltaire: un signe avant-coureur.' Lumen 13 (1994): 61-69.

8 Epître à Uranie (M.9:358;361). René Pomeau, pour sa part, qualifie la réaction de Voltaire de 'révolte': 'Mais, dans ce poème, la révolte a un accent qu'aucune oeuvre de Voltaire n'avait encore fait entendre.' La Religion de Voltaire, nouvelle éd. (Paris: Nizet, 1969) 113.

9 Geneviève Haroche-Bouzinac, Voltaire dans ses lettres de jeunesse (1711-1733), la formation d'un épistolier au XVIIIième siècle (Paris: Klincksieck, 1992) 333.

10 Lilian Willens, Voltaire's Comic Theatre: Composition, Conflict, and Critics, Studies on Voltaire and the Eighteenth Century 136 (1975): 49.

11 Marais, Journal cité dans M.2:243.

12 La Harpe 2:508.

13 Cf. Les petits vers à son intention, ainsi que l'allusion à sa modestie (M.2:282). 